\title{
Seasonal Evolution of Titan's Stratosphere Near the Poles
}

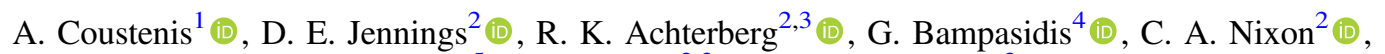 \\ P. Lavvas ${ }^{5}$ (D), V. Cottini ${ }^{2,3}$ (D), and F. M. Flasar ${ }^{2}$ (D) \\ ${ }^{1}$ Laboratoire d'Etudes Spatiales et d'Instrumentation en Astrophysique (LESIA), Observatoire de Paris, CNRS, UPMC Univ. Paris 06, \\ Univ. Paris-Diderot, 5, place Jules Janssen, F-92195 Meudon Cedex, France; athena.coustenis@obspm.fr \\ ${ }^{2}$ Planetary Systems Laboratory, Goddard Space Flight Center, Greenbelt, MD 20771, USA \\ ${ }^{3}$ Department of Astronomy, University of Maryland, College Park, MD 20742, USA \\ ${ }^{4}$ Faculty of Physics, National and Kapodistrian University of Athens, Panepistimioupolis, 15783 Zographos, Athens, Greece \\ ${ }^{5}$ GSMA, Reims Champagne-Ardenne, F-51687 Reims, France
}

\begin{abstract}
In this Letter, we report the monitoring of seasonal evolution near Titan's poles. We find Titan's south pole to exhibit since 2010 a strong temperature decrease and a dramatic enhancement of several trace species such as complex hydrocarbons and nitriles $\left(\mathrm{HC}_{3} \mathrm{~N}\right.$ and $\mathrm{C}_{6} \mathrm{H}_{6}$ in particular) previously only observed at high northern latitudes. This results from the seasonal change on Titan going from winter (2002) to summer (2017) in the north and, at the same time, the onset of winter in the south pole. During this transition period atmospheric components with longer chemical lifetimes linger in the north, undergoing slow photochemical destruction, while those with shorter lifetimes decrease and reappear in the south. An opposite effect was expected in the north, but not observed with certainty until now. We present here an analysis of high-resolution nadir spectra acquired by Cassini/Cassini Composite Infrared Spectrometer in the past years and describe the temperature and composition variations near Titan's poles. From 2013 until 2016, the northern polar region has shown a temperature increase of $10 \mathrm{~K}$, while the south has shown a more significant decrease (up to $25 \mathrm{~K}$ ) in a similar period of time. While the south polar region has been continuously enhanced since about 2012, the chemical content in the north is finally showing a clear depletion for most molecules only since 2015. This is indicative of a non-symmetrical response to the seasons in Titan's stratosphere that can set constraints on photochemical and GCM models.
\end{abstract}

Key words: infrared: planetary systems - planets and satellites: atmospheres - planets and satellites: composition planets and satellites: individual (Titan) - radiation mechanisms: thermal - radiative transfer

\section{Introduction}

The Cassini mission was in the Saturnian system observing, as a privileged target, Titan for about half of a Saturnian year. The last targeted Titan flyby was on 2017 April 22, followed by the start of the Cassini Grand Finale polar/proximal orbits the next day. Titan's northern hemisphere was in early winter when Cassini arrived in 2004 July and by the end of the extended mission, after 13 years in the system, it was just past northern summer solstice (2017). Within that time, we have had the opportunity to monitor Titan's atmosphere and surface response through two full seasons, including the same time as the Voyager encounter in 1980 November (Coustenis et al. 2013). Since the equinox in mid-2009, where both hemispheres received equal insolation, we have witnessed rapid and strong seasonal effects both in the atmosphere and on the surface of the satellite.

In particular for Titan's stratosphere, roughly between 50 and $400 \mathrm{~km}$ in altitude, variations in the thermal and chemical structure were reported from investigators analyzing spectra acquired by the Cassini Composite Infrared Spectrometer (CIRS; Flasar et al. 2004; Jennings et al. 2017). These investigations have shown that a large-scale reversal occurred in the single pole-to-pole circulation affecting the distribution of the atmospheric structure after the northern equinox in mid2009 , with the gases in the summer hemisphere showing upwelling while in the winter pole a corresponding subsidence was found (Achterberg et al. 2008, 2011; Bampasidis et al. 2012; Teanby et al. 2012). Whereas until 2011 a significant build-up of trace gases, haze, and condensates was still observed over the north pole (West et al. 2011, 2016; Jennings et al. 2012a, 2012b; Coustenis et al. 2013; Vinatier et al. 2015), toward the end of that period, the vortex and hotspot reported in Jennings et al. (2015) and Achterberg et al. (2008) had practically disappeared. In its place, similar features started to appear near the south pole, which have evolved to what we see today. In the absence of sunlight, gases that subsidize have accumulated since 2012 and become enhanced (e.g., Coustenis et al. 2016 and references within; Teanby et al. 2017), and a subset of those have been suggested to be causing the haze decrease found in the north and its subsequent increase in the south (Jennings et al. 2012b, 2015).

We have already published an analysis of spectra acquired by Cassini/CIRS at high resolution from 2010 onward, both in nadir and limb mode and at high and middle latitudes (Coustenis et al. 2013, 2016). In our 2016 work, we showed that the hydrocarbons found at high concentrations and longer lifetimes did not show significant latitudinal variability in the past years and until mid-2013. But in more recent dates, these molecules show a strong increase in the south. Notably, the $70^{\circ} \mathrm{S}$ and mid-latitude abundances show different trends indicating different dynamical processes at play inside and out of the polar vortex region extending down to $50^{\circ}$ in latitude. While most trace stratospheric gases in the $70^{\circ} \mathrm{N}$ data generally show only a small decreasing trend until 2014, the $70^{\circ} \mathrm{S}$ results on the contrary exhibit a strong enhancement after 2012. This argues for a fast and strong build-up of the gases in the southern pole as it goes deeper into the shadow during the 2013-2014 onset of winter, as predicted by models. The limb spectra also show variations in time with altitude, with, in 


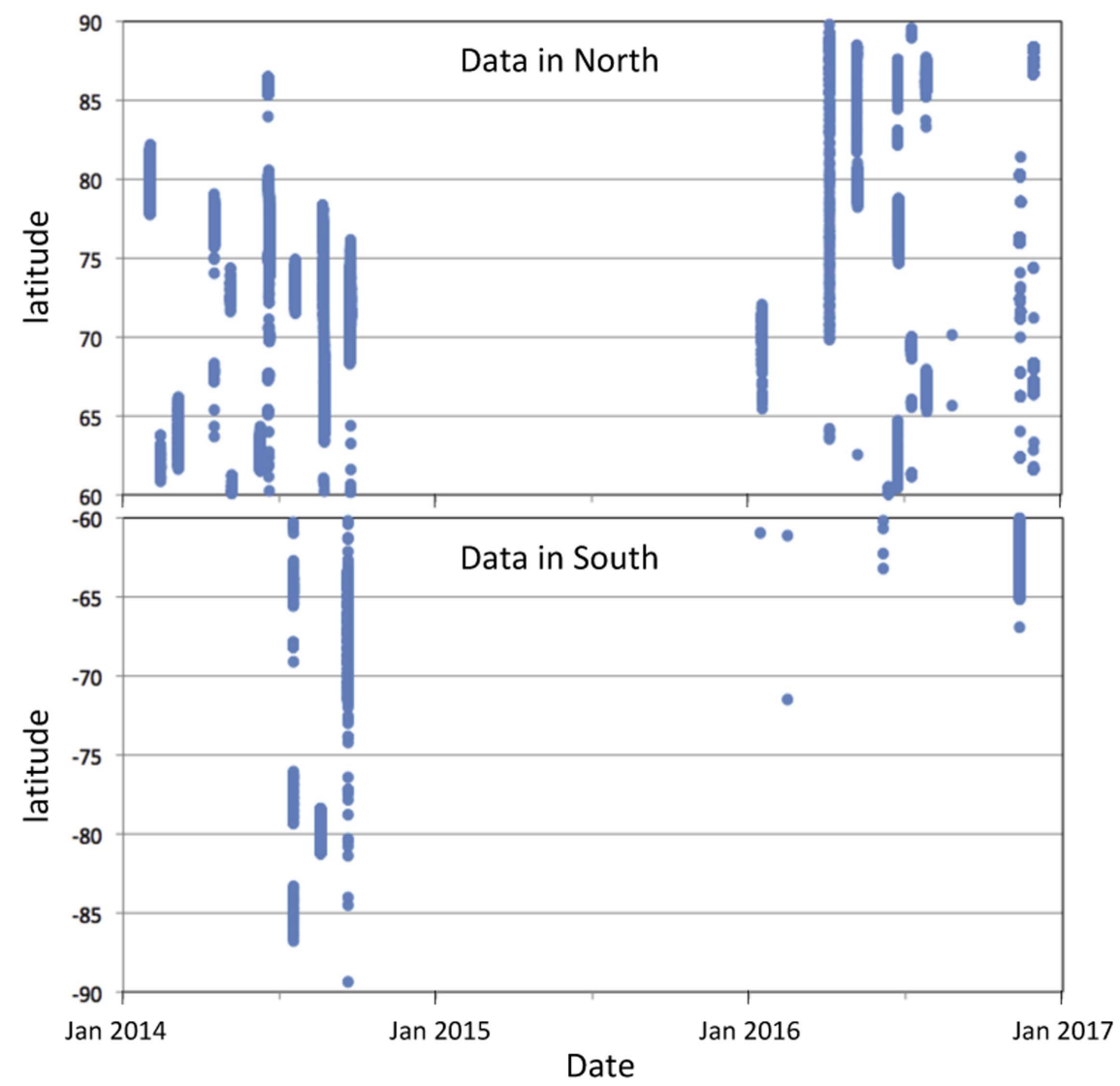

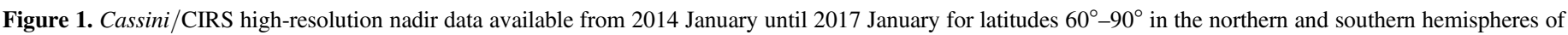

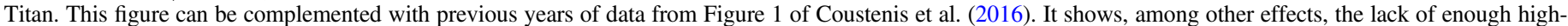
resolution CIRS observations of the north pole between the fall of 2014 and 2016 May and of the south pole from 2014 September onward.

particular, an area covering the whole northern hemisphere at altitudes between 400 and $500 \mathrm{~km}$ strongly depleted in gas and haze in early 2016, indicative of a complex dynamical effect yet to be identified (Vinatier et al. 2015; Teanby et al. 2017).

Here, we report on Cassini/CIRS observations of Titan's stratosphere at both high northern and southern latitudes (between $60^{\circ}$ and $90^{\circ}$ ) from 2012 to 2016 (where we have calibrated high-resolution data) to infer the behavior of the atmospheric temperature and chemical structure as a function of time and the results of such seasonal effects. In particular, this Letter follows the Coustenis et al. (2016) work on seasonal effects in the atmosphere, looking for the expected decrease in abundance of the northern pole constituents, not previously observed.

\section{Observations and Analysis}

For the purposes of this Letter, we exploited CIRS high spectral resolution $\left(0.5 \mathrm{~cm}^{-1}\right)$ data taken from 2012 to 2016 in the surface-intercepting nadir mode. CIRS is a Fourier transform spectrometer spanning $10-1500 \mathrm{~cm}^{-1}$ in three spectral channels or focal planes (FPs). We use here spectra from the far-infrared FP3 and FP4 that cover the $600-1500 \mathrm{~cm}^{-1}$ range (Jennings et al. 2017).

Figure 1 shows the high-resolution spectra of Titan's stratosphere acquired at high latitudes (from $60^{\circ}$ to $90^{\circ}$ ) from 2014 to 2017. The purpose of this figure is to expose unfortunate gaps in the observations for such high latitudes caused by the nature of Cassini's orbital geometry. Indeed, the north pole is not represented in the data between the fall of
2014 and 2016 May, and for the south pole we do not have enough high-resolution $\left(0.5 \mathrm{~cm}^{-1}\right)$ spectra available from fall 2014 onward. We then have to work with the existing data as far as temporal variations are concerned and to take into account these observational gaps.

Table 1 shows the spectra used in this work for inferring the time dependence of Titan's polar thermal and compositional structure in the stratosphere. With respect to previous studies, the data analyzed and interpreted here cover up to Titan Flyby 122 and essentially deal with recent data taken near the northern pole, where observations were missing until recently.

The selections that were already analyzed in Coustenis et al. (2016) are re-investigated here with spectra from an updated calibration. This concerns the 2012 June and 2013 May southern averages and the 2013 December-2014 September northern averages. All of the other selections in Table 1 are new (three new south pole averages: 2012 September, 2013 December, and 2014 September, and four new north pole averages: 2013 September, 2016 May, 2016 June, and 2016 August), so 7 out of a total of 12 data sets, are new. The new calibration provides a better sensitivity for line detection and the enhanced radiance in some of the parts of the FP3 spectrum allows for a more precise inference of the abundances and their variation with time for some of the weaker species. The difference in the results still remains within the 10\%-15\% range with respect to the abundances published in Coustenis et al. (2016).

The FOV of our CIRS observations was restricted to be entirely on Titan's disk and emission angles were limited to 
Table 1

FP3 Spectral Averages

\begin{tabular}{|c|c|c|c|c|c|c|}
\hline \multicolumn{7}{|c|}{ South Pole } \\
\hline Year & Month & Cassini Flyby & Latitudinal Bin & Number of Spectra & Airmass & $\operatorname{Ls}\left({ }^{\circ}\right)$ \\
\hline (*) 2012 & Jun & T84 & $65^{\circ} \mathrm{S}-75^{\circ} \mathrm{S}$ & 584 & 1.2 & 36 \\
\hline 2012 & Sep & T86 & $65^{\circ} \mathrm{S}-75^{\circ} \mathrm{S}$ & 878 & 1.5 & 38 \\
\hline (*) 2013 & May & T91 & $65^{\circ} \mathrm{S}-75^{\circ} \mathrm{S}$ & 78 & 1.3 & 45 \\
\hline 2013 & Dec & T97 & $75^{\circ} \mathrm{S}-85^{\circ} \mathrm{S}$ & 1077 & 1.1 & 52 \\
\hline 2014 & Sep & $\mathrm{T} 105$ & $70^{\circ} \mathrm{S}-80^{\circ} \mathrm{S}$ & 519 & 1.2 & 60 \\
\hline \multicolumn{7}{|c|}{ North Pole } \\
\hline Year & Month & Cassini Flyby & Latitudinal Bin & Number of Spectra & Airmass & $\operatorname{Ls}\left({ }^{\circ}\right)$ \\
\hline 2013 & Sep & T94 & $65^{\circ} \mathrm{N}-75^{\circ} \mathrm{N}$ & 544 & 1.3 & 49 \\
\hline (*) 2013 & Dec & T97 & $70^{\circ} \mathrm{N}-80^{\circ} \mathrm{N}$ & 1713 & 1 & 52 \\
\hline$\left(^{*}\right) 2014$ & Feb & T98 & $70^{\circ} \mathrm{N}-80^{\circ} \mathrm{N}$ & 1488 & 1 & 54 \\
\hline (*) 2014 & Sep & $\mathrm{T} 105$ & $70^{\circ} \mathrm{N}-80^{\circ} \mathrm{N}$ & 541 & 1.8 & 60 \\
\hline 2016 & May & $\mathrm{T} 119$ & $65^{\circ} \mathrm{N}-85^{\circ} \mathrm{N}$ & 780 & 1.5 & 78 \\
\hline 2016 & Jun & $\mathrm{T} 120$ & $65^{\circ} \mathrm{N}-85^{\circ} \mathrm{N}$ & 725 & 1.5 & 79 \\
\hline 2016 & Aug & $\mathrm{T} 122$ & $65^{\circ} \mathrm{N}-85^{\circ} \mathrm{N}$ & 1072 & 1.3 & 82 \\
\hline
\end{tabular}

FP4 Spectral Averages

\begin{tabular}{|c|c|c|c|c|c|c|}
\hline \multicolumn{7}{|c|}{ South Pole } \\
\hline Year & Month & Cassini Flyby & Latitudinal Bin & Number of Spectra & Airmass & $\operatorname{Ls}\left({ }^{\circ}\right)$ \\
\hline (*) 2012 & Jun & T84 & $65^{\circ} \mathrm{S}-75^{\circ} \mathrm{S}$ & 356 & 1.2 & 36 \\
\hline 2012 & Sep & T86 & $65^{\circ} \mathrm{S}-75^{\circ} \mathrm{S}$ & 742 & 1.4 & 38 \\
\hline (*) 2013 & May & T91 & $65^{\circ} \mathrm{S}-75^{\circ} \mathrm{S}$ & 16 & 1.6 & 45 \\
\hline 2013 & Dec & T97 & $75^{\circ} \mathrm{S}-85^{\circ} \mathrm{S}$ & 802 & 1.1 & 52 \\
\hline 2014 & Sep & T105 & $70^{\circ} \mathrm{S}-80^{\circ} \mathrm{S}$ & 1187 & 1.2 & 60 \\
\hline \multicolumn{7}{|c|}{ North Pole } \\
\hline Year & Month & Cassini Flyby & Latitudinal Bin & Number of Spectra & Airmass & $\operatorname{Ls}\left({ }^{\circ}\right)$ \\
\hline 2013 & Sep & T94 & $65^{\circ} \mathrm{N}-75^{\circ} \mathrm{N}$ & 740 & 1.3 & 49 \\
\hline (*) 2013 & Dec & T97 & $70^{\circ} \mathrm{N}-80^{\circ} \mathrm{N}$ & 854 & 1.1 & 52 \\
\hline (*) 2014 & Feb & T98 & $70^{\circ} \mathrm{N}-80^{\circ} \mathrm{N}$ & 1397 & 1.2 & 54 \\
\hline (*) 2014 & Sep & $\mathrm{T} 105$ & $70^{\circ} \mathrm{N}-80^{\circ} \mathrm{N}$ & 783 & 1.8 & 60 \\
\hline 2016 & May & $\mathrm{T} 119$ & $65^{\circ} \mathrm{N}-85^{\circ} \mathrm{N}$ & 957 & 1.4 & 78 \\
\hline 2016 & Jun & $\mathrm{T} 120$ & $65^{\circ} \mathrm{N}-85^{\circ} \mathrm{N}$ & 1190 & 1.3 & 79 \\
\hline 2016 & Aug & $\mathrm{T} 122$ & $65^{\circ} \mathrm{N}-85^{\circ} \mathrm{N}$ & 735 & 1.2 & 82 \\
\hline
\end{tabular}

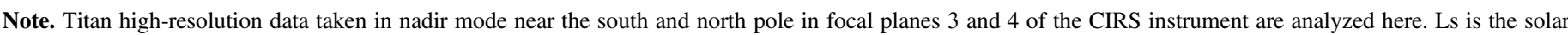

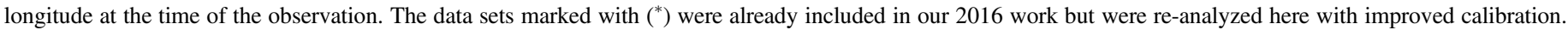

$0^{\circ}-65^{\circ}$ to avoid the need for large atmospheric corrections. To secure good sensitivity we only use FP3 averages with more than 500 spectra in a bin (with one exception in 2013 May at high southern latitudes) and with as many as about 1100 spectra in some cases. Available spectra within each time period were zonally averaged in $10^{\circ}$ latitude bins for the south and $10^{\circ}$ or $20^{\circ}$ latitude bins for the north that covered latitudes within the $65^{\circ} \mathrm{S}-85^{\circ} \mathrm{S}$ range. The averages depend on the data available for a given date/flyby within a latitude range and aim at improving the signal-to-noise ratio (which is at worst 10 and at best around 50 on average; depending on the selection, the lower values of the $\mathrm{S} / \mathrm{N}$ apply mostly to the southern data). Thus, in some cases, we had to extend the latitude range toward lower or higher latitudes, but we checked in our contribution functions that the atmospheric range probed remains in the same pressure levels. For purposes of clarity, all of these spectral averages are marked "SP" in the plots and discussion hereafter. We apply the same process for the data near the north pole and we mark their averages by "NP".

We use a monochromatic radiative transfer code adapted to Titan's atmosphere (ARTT; Coustenis et al. 2010; Bampasidis et al. 2012), and we extract the temperature profile from the best fit of the $7.7 \mu \mathrm{m}$ methane band in FP4 (Figure 2 shows some examples of fitting the methane $v_{4}$ band in the $1250-1350 \mathrm{~cm}^{-1}$ region). Our model is based on a $\mathrm{CH}_{4}$ abundance of $1.5 \%$ through the stratosphere and increasing below the cold trap to about $5 \%$ at the surface, in agreement with CIRS and with Huygens Gas Chromatograph Mass Spectrometer (GCMS) measurements (Flasar et al. 2004; Niemann et al. 2010). The temperature profile is originally set to the one measured at altitudes $0-147 \mathrm{~km}$ by the Huygens Atmospheric Structure Instrument (HASI; Fulchignoni et al. 2005). Adjustments were applied to the HASI profile to extend in altitude and to account for latitude dependences and seasonal variations in temperature as found by CIRS emission values in the $7.7 \mu \mathrm{m} \mathrm{CH} 4$ band and from lower-resolution observations (Coustenis et al. 2007, 2010, 2016; Achterberg et al. 2008, 2011; Bampasidis et al. 2012). For more details on the method see the literature.

We then implement the temperature profile in our radiative transfer code and solve for the opacity in the rest of the spectrum retrieving the abundances of trace gases and their 

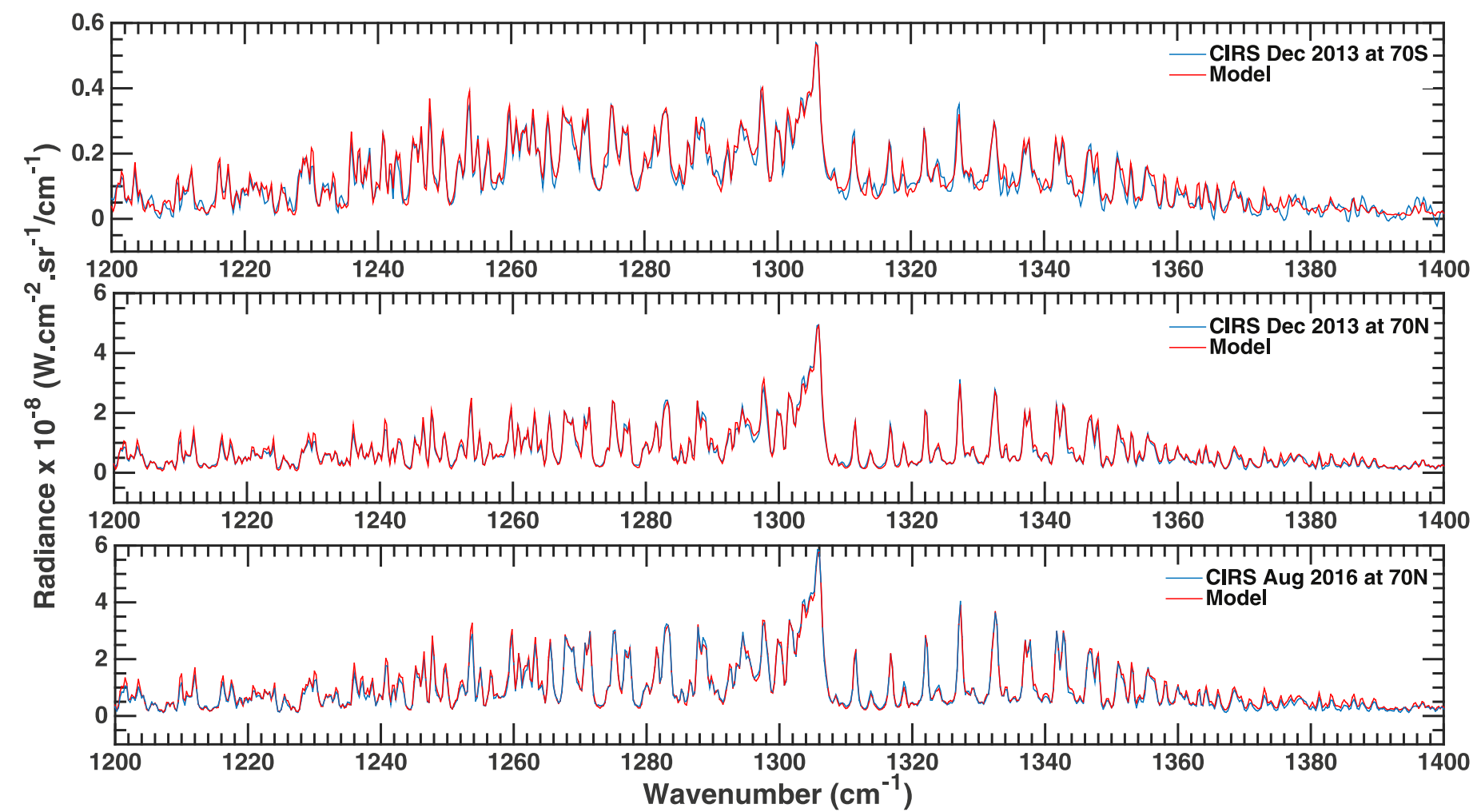

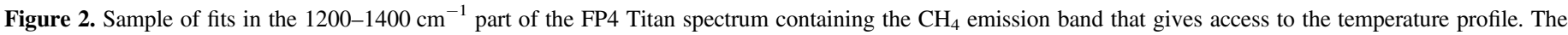

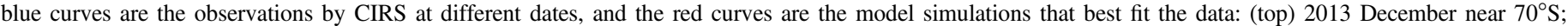
(middle) 2013 December near $70^{\circ} \mathrm{N}$; (bottom) 2016 August near $70^{\circ} \mathrm{N}$.

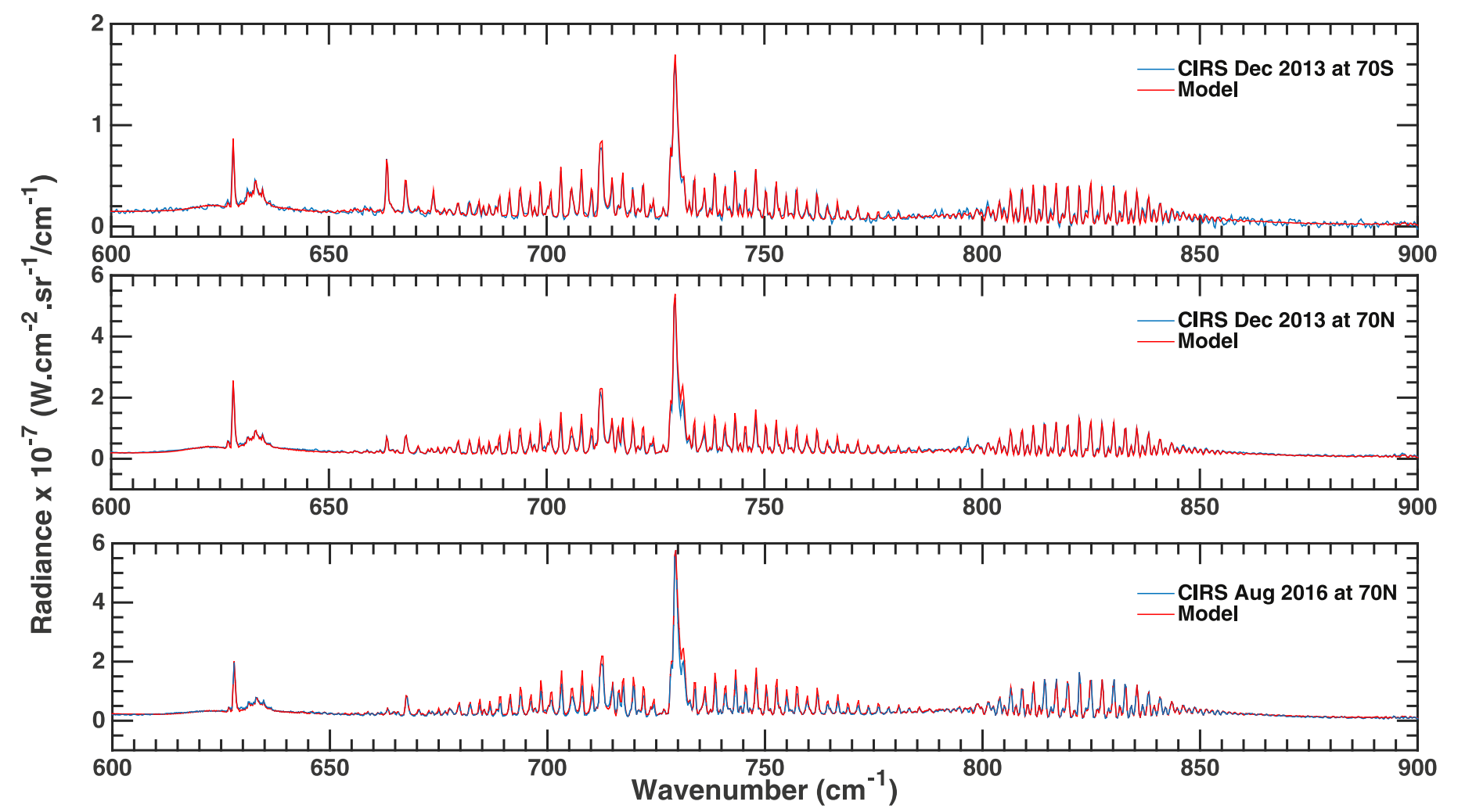

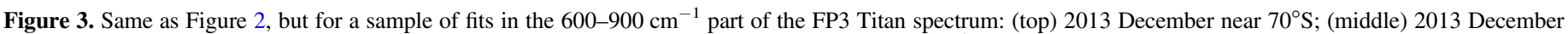

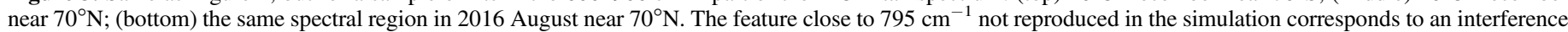
pixel in the data that was not completely suppressed in some cases. 
isotopes in the FP3 region of the CIRS spectra. Figure 3 shows some of our fits in the $600-900 \mathrm{~cm}^{-1}$ range where we observe the emission of several gaseous bands (e.g., $\mathrm{HCN}, \mathrm{C}_{2} \mathrm{H}_{2}, \mathrm{C}_{3} \mathrm{H}_{8}$, $\mathrm{C}_{2} \mathrm{H}_{6}$ ). Our spectra are taken in the nadir mode so we do not have information on the altitude dependence of the mixing ratios, We apply constant-with-height vertical profiles from the higher levels of the atmosphere (around $400 \mathrm{~km}$ in altitude) down to the condensation level and then following the saturation curve. This assumption is essentially valid for all of the molecules considered here, due to their weak emission bands, except for $\mathrm{C}_{2} \mathrm{H}_{2}$ whose abundance variations with height should be taken into account. Our method for inferring the acetylene mixing ratio values by making use of weighted averages over the $\mathrm{P}, \mathrm{Q}$, and $\mathrm{R}$ branches of the $729 \mathrm{~cm}^{-1}$ band and by testing vertical profiles is described in detail in Section 2 of Bampasidis et al. (2012).

\section{Results and Discussion}

In our recent work (Coustenis et al. 2016), we inferred the temperature profiles and the chemical composition at different dates from 2010 and until 2014 for high northern and southern latitudes (at and beyond $50^{\circ} \mathrm{N}$ and $50^{\circ} \mathrm{S}$ ).

We had already shown then that as the southern hemisphere moved into winter after 2010, large temperature variations were observed near the south pole $\left(70^{\circ} \mathrm{S}\right)$ in the stratosphere (from 0.1 down to 1 mbar pressure levels). Indeed, while a moderate warming is observed in the summer-entering north for the middle and high northern latitudes, a decrease of about $10-15 \mathrm{~K}$ in temperature was measured already for the $50^{\circ} \mathrm{S}$ latitudes. A more spectacular drop in temperature by as much as $25 \mathrm{~K}$ at $70^{\circ} \mathrm{S}$ was measured from 2012 to 2014 . These temperature variations were accompanied by a strong enhancement of chemical compounds in the south polar region, while the north failed to show the opposite effect, which indicated a non-symmetrical reaction to the seasonal influence for each pole. We explore here more recent dates seeking to determine longer-term seasonal effects near Titan's poles.

\subsection{Thermal Structure Variations in the Stratosphere Near the Poles from 2012 to 2016}

In Figure 5, we show the stratospheric temperature on Titan at $70^{\circ} \mathrm{S}$ with solid lines for different years and with different colors from 2012 to 2014 and at around $70^{\circ} \mathrm{N}$ with dashed lines for different dates from 2013 to 2016. Our contribution functions indicate that the emission observed in the $7.7 \mu \mathrm{m}$ methane band originates from levels 0.01-20 mbar (70-400 km in altitude), with a peak around 0.1-1 mbar.

We note that we do not have nadir high-resolution CIRS data for the south pole after mid-2014 (Figure 1). Comparing the earlier dates in the northern polar region (light and dark blue and red dashed lines) with the other dates, we find relatively moderate changes: the new spectral averages from 2016 observations show that the temperature increased by $10 \mathrm{~K}$ from 2013 to 2016. The north pole then took three years to gain $10 \mathrm{~K}$, while the south pole lost 2.5 times as much in only two years. There does not therefore appear to be a direct symmetry in the thermal response from each pole on Titan in the stratosphere in terms of temperature. In particular, a $6 \mathrm{~K}$ warming is found in the north during the period from 2014 February to September, whereas during approximately the same period of time, the southern stratospheric temperature

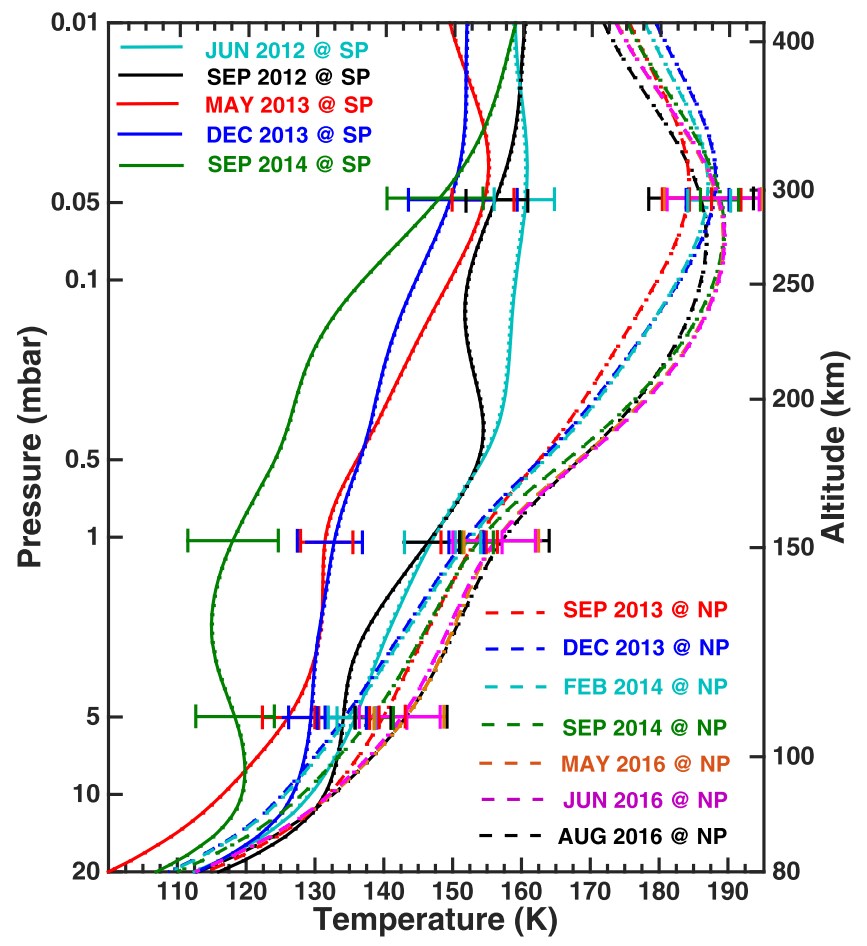

Figure 4. Temperature evolution in Titan's stratosphere near the poles from 2012 to 2016. The southern polar profiles are shown with solid lines, while the north pole profiles are depicted with dashed lines and the different dates are indicated with different colors. $3 \sigma$ uncertainties are shown.

dropped by an additional $12-15 \mathrm{~K}$ near $0.5 \mathrm{mbar}$, totaling up to a $25 \mathrm{~K}$ loss in a little more than two years. From 2014 to 2016, the north pole has gained another $2 \mathrm{~K}$. The real increase in temperature in the north is actually significant only after 2014 September (Figure 4). The decrease in the stratospheric temperature in the south began as early as late 2011 (Coustenis et al. 2016). Our temperature profile for 2014 September is quite compatible with the results of limb observations in 2015 March by Teanby et al. (2017).

We then report here that the north polar region's temperature is beginning to show a reaction to the increasing sunlight as the north moves into summer, but with a three-year delay with respect to the high southern latitudes. This should be accompanied by a decrease in radiative emitters, as already observed for latitudes around for $50^{\circ} \mathrm{N}$, a latitude located at the border of the northern vortex, which was shown to be weakening in the past years (Teanby et al. 2012; Jennings et al. 2015).

\subsection{Chemical Composition Changes in the Stratosphere Near the Poles from 2012 to 2016}

We have demonstrated that as the southern hemisphere moved into winter after 2010, strong temperature and composition variations are found essentially in the south pole. The drop in temperature over the south pole is associated with a strong enhancement in chemical content near the south pole. We thus witness in Titan's south pole the appearance and positive evolution of several trace species, such as $\mathrm{HC}_{3} \mathrm{~N}$ and $\mathrm{C}_{6} \mathrm{H}_{6}$, previously observed only at high northern latitudes. This is due to the evolution of Titan's seasons, going from northern winter in 2002 to advanced northern spring in 2012 with the opposite effect in the south pole as it moves into winter. During that time, Titan's main atmospheric circulation cell reverses 

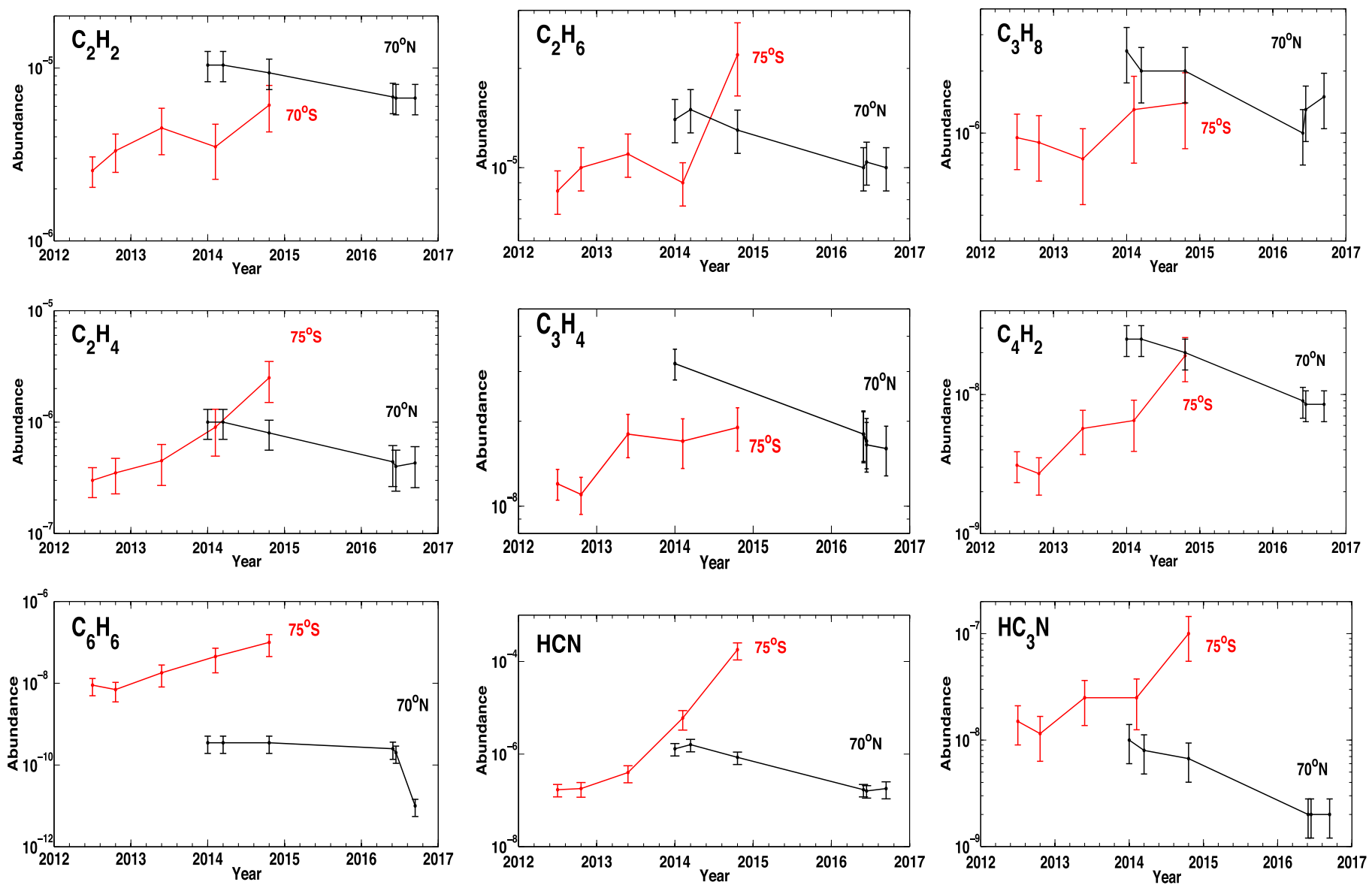

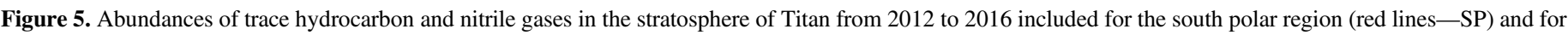
the north polar region (black lines-NP). $3 \sigma$ error bars are indicated.

and models predict that it channels newly produced photochemical species from mid-latitudes toward the south pole.

Figure 5 shows our results for the abundances of some of the minor gaseous components near both poles, with a maximum of the contribution functions in the $0.1-10$ mbar range $(90-250 \mathrm{~km})$. While subtle changes were seen for middle southern latitudes near $50^{\circ} \mathrm{S}$ (Coustenis et al. 2016), the red lines representing the south polar latitudes around $70^{\circ} \mathrm{S}$ in Figure 5 (indicated as SP) show an increase in the abundances by one or several orders of magnitude for many gases. For all of the trace molecules shown in this figure, the higher southern latitudes have become as or even more laden in organics than the north by late 2014. We thus note that the SP content was considerably increased from late 2010 until 2014 September, and in particular since 2012 by a factor of 2.5 for $\mathrm{C}_{3} \mathrm{H}_{4}$ and almost an order of magnitude for $\mathrm{C}_{4} \mathrm{H}_{2}$. As was already shown in Coustenis et al. (2016), the increase is even more dramatic at SP (by at least three orders of magnitude) for $\mathrm{C}_{6} \mathrm{H}_{6}, \mathrm{HCN}$, and $\mathrm{HC}_{3} \mathrm{~N}$. This enhancement has been noticed since late 2010 for $\mathrm{HCN}$ and $\mathrm{HC}_{3} \mathrm{~N}$ and since late 2011 for $\mathrm{C}_{6} \mathrm{H}_{6}$ (it should be noted that we only have upper limits prior to that date for these molecules; see Coustenis et al. 2016), and these species were significantly more enhanced at SP than at NP by late 2014 (by more than one order of magnitude for $\mathrm{HCN}$ and $\mathrm{C}_{6} \mathrm{H}_{6}$ and by two orders of magnitude for $\mathrm{HC}_{3} \mathrm{~N}$ ).

When compared to the findings of Teanby et al. (2017) for the southern polar regions from nadir data, we find remarkable agreement for all the molecules in the 2014-2015 period, with the exception of the nitriles $\left(\mathrm{HCN}\right.$ and $\left.\mathrm{HC}_{3} \mathrm{~N}\right)$ where our results indicate higher abundances already by about an order of magnitude. That could be due to the difference in spectral resolution (Teanby et al. use lower-resolution data), to the altitude ranges probed, and to the exact time periods. But all in all, our results are consistent.

The atmospheric constituents that were transported to the northern hemisphere during the previous season remain concentrated there around the north pole (indicated as NP in Figure 5) and are subjected to a slow photochemical destruction. Following the northern winter, those among the species with longer chemical lifetimes are expected to remain in the north for a longer time, while those with shorter lifetimes should be removed, reappearing in the south (Coustenis et al. 2016). The purpose of our investigation here is to determine how quick or slow this process is.

We do not have any high-resolution nadir data at $70^{\circ} \mathrm{N}$ before 2014, so we cannot infer the evolution of the abundances of these species prior to the past year, but they do not seem to have changed much since their values reported in Vinatier et al. (2015) from limb data in 2011 (their Figure 8). With that caveat in mind, our radiative transfer results show that the high northern latitudes (at about $20^{\circ}$ from the pole) only became significantly depleted in trace gases after 2015 (Figure 5). No significant difference is found for $\mathrm{CO}_{2}$ (not shown in Figure 5), as in previous studies. A limited drop in mixing ratios within the error bars is measured for the other most abundant molecules resisting the photolysis (such as 
$\mathrm{C}_{3} \mathrm{H}_{8}, \mathrm{C}_{2} \mathrm{H}_{6}, \mathrm{C}_{2} \mathrm{H}_{2}$, or $\mathrm{C}_{2} \mathrm{H}_{4}$ ), given the uncertainties. However, the trend for a drop in content for these molecules is attested by the consistently lower values found for these species in all 2016 spectral averages with respect to 2014. More remarkable decreases in abundance at NP are found for $\mathrm{C}_{3} \mathrm{H}_{4}$ and $\mathrm{C}_{4} \mathrm{H}_{2}$ (by $50 \%-70 \%) ; 25 \%-35 \%$ decreases are found in the mixing ratios of $\mathrm{C}_{6} \mathrm{H}_{6}, \mathrm{HCN}$, and $\mathrm{HC}_{3} \mathrm{~N}$.

The southern and northern polar values seem to cross (become similar) at around late 2014 for the hydrocarbons and earlier (at the beginning of 2014) for the nitriles.

\section{Conclusions}

We have evaluated the temperature and chemical composition variations in the stratosphere near Titan's poles from 2012 to 2017. The retrieved thermal profiles show that there is a three-year lag in the northern response with respect to the south.

Following the advent of winter in Titan's north, species with longer chemical lifetimes linger in the north while those with shorter lifetimes decrease and reappear in the south. Where we have data, we searched for signatures of weak stratospheric gases in large spectral averages around the poles and determined their abundances. The results show that the southern pole of Titan became hugely enhanced in trace gases after 2012. The northern composition decrease set in only about three years after the south enhancement had begun (roughly 2015 versus 2012).

The cause of these variations in abundance and temperature could be spatial changes (due to Titan's inclination) in the energy input to Titan's atmosphere, which acts as a driver for changes in the advection patterns, circulation, meteorology phenomena, and other physical processes. Those in turn lead to a stronger variability in the latitudinal abundances of the photochemical species after some time.

Among the most recent GCM works for Titan's atmosphere (Newman et al. 2011; Lebonnois et al. 2012; Lora et al. 2015), only the Institut Pierre-Simon-Laplace (IPSL) Titan GCM is coupled to atmospheric photochemistry, but no complete study of the seasonal variations of atmospheric composition has been published yet. According to a preliminary study (Lebonnois \& Vinatier 2013), the decrease of modeled trace gas abundances in the winter polar region at pressures around 1-10 mbar starts as soon as spring equinox, and the enrichment of the opposite polar region begins as soon as the autumn equinox. In this GCM, both variations are synchronized together and with the equinox, which is not what has been observed with the CIRS data set, probably indicating that more work is needed to refine the model. This work then helps to set strong constraints on photochemistry and circulation models that need to account for the asymmetry found in the poles' behavior.
The lack of observations of Titan's atmosphere near the poles over long periods of time begs for a return to Titan with a dedicated mission, after the end of the highly successful Cassini-Huygens mission in 2017 September. Benefiting from the whole pool of CIRS spectra until the end of the mission should allow us to better constrain the degree of complexity attained by the chemistry in Titan's stratosphere and to search for new molecules.

The authors acknowledge support from NASA's Cassini mission and Cassini data Analysis program. Our thanks to Sebastien Lebonnois for interesting discussions and for insights on the current status of GCM models.

\section{ORCID iDs}

A. Coustenis (iD https://orcid.org/0000-0003-3414-3491

D. E. Jennings (1D https://orcid.org/0000-0003-1340-754X

R. K. Achterberg (i) https://orcid.org/0000-0002-7643-7626

G. Bampasidis (1) https://orcid.org/0000-0002-0109-7823

C. A. Nixon (iD https://orcid.org/0000-0001-9540-9121

P. Lavvas (i) https://orcid.org/0000-0002-5360-3660

V. Cottini (iD https://orcid.org/0000-0003-0839-5855

F. M. Flasar (ib https://orcid.org/0000-0001-7186-5181

\section{References}

Achterberg, R. K., Conrath, B. J., Gierasch, P. J., Flasar, F. M., \& Nixon, C. A 2008, Icar, 194, 263

Achterberg, R. K., Gierasch, P. J., Conrath, B. J., Flasar, F. M., \& Nixon, C. A. 2011, Icar, 211, 686

Bampasidis, G., Coustenis, A., Achterberg, R. K., et al. 2012, ApJ, 760, 144 Coustenis, A., Achterberg, R. K., Conrath, B. J., et al. 2007, Icar, 189, 35 Coustenis, A., Bampasidis, G., Achterberg, R. K., et al. 2013, ApJ, 779, 177 Coustenis, A., Jennings, D. E., Achterberg, R. K., et al. 2016, Icar, 270, 409 Coustenis, A., Jennings, D. E., Nixon, C. A., et al. 2010, Icar, 207, 461 Flasar, F. M., Kunde, V. G., Abbas, M. M., et al. 2004, SSRv, 115, 169 Fulchignoni, M., Ferri, F., Angrilli, F., et al. 2005, Natur, 438, 785

Jennings, D. E., Achterberg, R. K., Cottini, V., et al. 2015, ApJL, 804, L34 Jennings, D. E., Anderson, C. M., Samuelson, R. E., et al. 2012a, ApJL, 754, L3 Jennings, D. E., Anderson, C. M., Samuelson, R. E., et al. 2012b, ApJL, 761, L15

Jennings, D. E., Flasar, F. M., Kunde, V. G., et al. 2017, ApOpt, 56, 5274

Lebonnois, S., Burgalat, J., Rannou, P., \& Charnay, B. 2012, Icar, 218, 707 Lebonnois, S., \& Vinatier, S. 2013, AGUFM, 2013, P53C1869L

Lora, J. M., Lunine, J. I., \& Russell, J. L. 2015, Icar, 250, 516

Newman, C. E., Lee, C., Lian, Y., Richardson, M., \& Toigo, A. D. 2011, Icar, 213,636

Niemann, H. B., Atreya, S. K., Demick, J. E., et al. 2010, JGR, 115, E12006 Teanby, N. A., Bézard, B., Vinatier, S., et al. 2017, NatCo, 8, 1586

Teanby, N. A., Irwin, P. G. J., Nixon, C. A., et al. 2012, Natur, 491, 732 Vinatier, S., Beìzard, B., Lebonnois, S., et al. 2015, Icar, 250, 95

West, R. A., Balloch, J., Dumont, P., et al. 2011, GeoRL, 38, L06204

West, R. A., Del Genio, A. D., Barbara, J. M., et al. 2016, Icar, 270, 399 